\begin{tabular}{c} 
Volume and Issues Obtainable at Center for Sustainability Research and Consultancy \\
Journal of Business and Social Review in Emerging Economies \\
ISSN: 2519-089X \& ISSN (E): 2519-0326 \\
Volume 7: Issue 2 June 2021 \\
CSRC \\
Journal homepage: $\underline{\text { www.publishing.globalcsrc.org/jbsee }}$ \\
\hline
\end{tabular}

\title{
Excavating Future Challenges: An Analysis of Health Systems of One Hundred-Six Countries
}

*Abdul Basit, Lahore Institute of Science \& Technology, Lahore, Pakistan

Waheed Asghar, Director in Technical Education and Vocational Training Authority, (Government of the Punjab), Lahore, Pakistan

Abdul Aziz Khan Niazi, Institute of Business and Management, University of Engineering and Technology, Lahore, Pakistan

Tehmina Fiaz Qazi, ${ }^{4}$ Hailey College of Banking and Finance, University of the Punjab, Lahore, Pakistan

*Corresponding author's email: abasit_shahbaz@yahoo.com

\begin{tabular}{l} 
ARTICLE DETAILS \\
\hline History \\
Revised format: May 2021 \\
Available Online: Jun 202 \\
\hline Keywords \\
Countries, Future \\
challenges, GRA, Health \\
risk, Health system.
\end{tabular}

JEL Classification $M 0, M 1$

\section{OPEN ACCESS}

\section{ABSTRACT}

Purpose: Health system of a country is backbone of economy. It has fundamental importance in sustainable development of a country. Aim of this article is to excavate future challenges to health system of selected 106 countries.

Design/Methodology/Approach: It is a country level comparative analysis of health risk factors. Design of the study includes review of literature, data extraction and analysis. The cross-sectional secondary data has been drawn from website of World Development Indicators (WDI) 2020. Grey relational analysis is used as technique of investigation.

Findings: Results show that majorly, member countries of Organization for Economic Co-operation and Development (OECD) have exceptionally high grey relational grade, therefore, are considered to be countries having less future health risks, whereas, Southern African Development Community (SADC) have exceptionally low grey relational grade, therefore, have high future health risk.

Implications/Originality/Value: It is a unique study using different dataset and methods that provides valuable insights to political governments, researchers and health system managers.

(C) 2021 The authors, under a Creative Commons AttributionNonCommercial 4.0

\footnotetext{
Recommended citation: Basit, A., Asghar, W., Niazi, A. A. K. and Qazi, T. F. (2021). Excavating Future Challenges: An Analysis of Health Systems of One Hundred-Six Countries. Journal of Business and Social Review in Emerging Economies, 7 (2), 219-230.
}

\section{Introduction}

Health system of a country is considered as one of the core systems of an economy. The political governments are concerned with health systems. The health systems have been exposed to different risks from time to time. In recent times this system has faced shocks of Covid-19 
pandemic. Health systems have been shattered in most of the countries during this pandemic period. This discipline is rich in research and there is flood of research articles in context of cronavirus now a days. Evaluation of health system has gained imperative importance and it is ever high on the agenda of research. Country level evaluation of health systems is also vital to study. A comparative study of countries' level health systems is also that of fundmental importance. In order to set out the context of the study, it is pertinent to report some resent and relevant studies herein viz challenges of healthcare service in Italy (Armocida et al., 2020), environmental, social and health issues of Mexican health system (de León-Martínez et al., 2020), Bulgarian health system challenges (Dzhafer \& Papathanasiou, 2020), Taiwanese health system resilience (Hsieh, 2020), health risk factors: the case of Luxembourg, France (Mussard and Alperin, 2021), Brazilian health system risks (Requia et al., 2020), challenges of health system of sub-Saharan Africa (Roder-DeWan, 2020) and health risk management behaviors of Japanese university students (Yamakawa et al., 2019). Global health programs are aimed to remove social, cultural, and logistical barriers to fill the gap in health disparities and health related knowledge between developed and developing countries. Health matrices and research are essential for planning, policy making, programming and accountability. There is a severe need of high quality health information to make the research gaps clearer. Despite of plenty of research there is still room for systemic studies. Therefore, this study has aimed to evaluate the future challenges to health system of 106 countries of the world. It is also aimed to rank the countries on basis of level of possible challenges to health system of a country. Further, it aims to classify and discuss the results bloc wise. To achieve these objectives Grey Relational Analysis (GRA) is opted as research methodology. Remaining article is arranged as literature review, methodology, data analysis, results \& discussion and conclusion.

\section{Literature Review}

Contemporary literature is necessary to sum up the up to date research findings of the phenomenon in hand. We explored renowned databases of research such as Ebrary, Elsevier (ScienceDirect), Emerald, Springerlink, Taylor \& Francis Journals, Wiley-Blackwell Journals etc. and reviewed sufficient relevant studies. Few of which qualify to be reported here in order to establish hard ground of the study in hand e.g. studies like: safety and risk management including: pharmacy role in health system of Colombia (Amariles et al., 2020), Mount Sinai health system in US (Buckley et al., 2020), health services system of Hubei, China (Chen et al., 2020), examine psychosocial risk factors in public workplace in Denmark (Dahler-Larsen et al., 2020), vexed Greek health care system (Kapetanakis et al., 2020), resilience of Spanish health system (Legido-Quigley et al., 2020), strained Latin American health care system (Navarro et al., 2020), challenges facing in health and social care planning in Spain (Queralt-Tomas et al., 2019) and safety risk management and occupational health of waste management firms in Portugal (Ramos et al., 2020). Aghdam et al. (2020) carried a detailed study on effective usage of Internet of Things (IoT) to facilitate the paramedical staff and health workers and also to identify the major trends and challenges facing the healthcare. Bolnick et al. (2020) revealed that high spending on health care ensures the control risk exposure, reduces health burden and cost. Briggs et al. (2020) reported some key challenges and opportunities facing by global health policy. Cheng et al. (2019) concluded that agriculture soil contamination is posing significant risks to human health and food safety. Dahler-Larsen et al. (2020) stressed that occupational health and safety risk management estimate action fairly well against psychological risks as compare to physical risks. de León-Martínez et al. (2020) analyzed the environmental (contaminants from biomass burning), social (lack of water access, limited internet access, return of aboriginal people to their communities, language barriers), and health risk factors (hypertension, chronic respiratory disease, diabetes, respiratory tract infections) in context of COVID-19 to mitigate its impact on society at large. Gao et al. (2020) stated that circumjacent air pollution is negatively associated with health risk and strategy must be devised to mitigate this risk. Mhango et al. (2020) reported some key factors (acquaintance to infected patients, poor 
infection control mechanism, personal protective equipment shortage and preexistent medical facility) as COVID-19 risk factors among health workers. Dash (2019) conducted a systematic review of literature to exhume the vital challenges and future directions of big data analytics and its impact on healthcare. He further argued that while processing the large data; data security and management is the major challenge being faced by data analytics. Mussard and Alperin (2021) examined health indicators of two-parameter family linking one risk factor and a health dimensions by taking four factors including parents longevity, financial condition during childhood, parental education and parents nationality and found that parents education and migration background are the most exogenous risk factors contributing in socio-economic health inequality. Najera and Ortega-Avila (2020) affirmed that COVID-19 mortality risk rises significantly in patients in form of diabetes, obesity, cardiovascular disease or hypertension, however, for patients with same profile, the death risk dramatically different. Ramos et al. (2020) bolstered that implementation of integrated management system has proved significant success in improvement of occupational health and safety risk management process in businesses. Rohat et al. (2019) examined the European future heat related health issues and found capricious socioeconomic development sustainability as the major causes. Scharpf et al. (2020) conducted a systematic review to study the mental health of refugees associated with risk protection and buttressed that challenges of relocation, violent conflict and flight cause severe damage on mental health and well-being. Xu et al. (2021) stated that there is a direct and significant positive relationship between health risks and economic development. Yadav et al. (2021) argued that contaminants of emerging concerns in soil, water and air adversely effect on environment and risks of human health. Yang et al. (2020) proclaimed that psychological risk management soft policies rank higher than hard policies; furthermore national level policies score more than at local level in China. It is further explained that China is still behind by adopting the safety related psychological health policies as compare to Europe and Australia. Yamakawa et al. (2019) highlighted that parental occupation and travel experience of overseas offer a pertinent insights of promoting health risk management. Zaidi et al. (2021) identified and ranked eleven challenges hampering sustainable public health sector of Pakistan and found government rules \& regulations and senior management commitment are the major obstacles in implementing it. Zhang and Mohandes (2020) carried a comprehensive study on occupational health and safety and proposed a framework contributing in: identifying green-go requirements, ascertaining list of safety risks associated with green-oriented requisite through Delphi and Znumbers method, assessing safety risks by way of proposing five-layers strategy, estimating green-oriented safety risks magnitude using Z-number based algorithm and providing the measures to overcome/control the greenoriented safety risks. The review of literature pinpoints that there is dearth of country level comparative studies of health systems particularly based on multiple criteria. Hardly any study can be found that compares hundreds of the countries based on a multitude of future challenges to be faced by different countries. Neither the comparative studies nor the comprehensive methodologies have been witnessed from within the literature. Therefore, to develop a comprehensive framework, apply some composite methodology and comparing the country level future risks to health systems is something inevitable to enrich the contemporary literature.

Theoretical Framework: Theoretical framework fixes perimeters of scope of research. Data, method, tentative solution to the problem are signposted by the framework of the study. We have adopted the framework from World Development Indicator (WDI) 2020. Variables, operational definitions, unit of measurement and data set are taken from WDI-2020.

Table 1: Health Risk Factors

\begin{tabular}{cccc}
\hline Code & Health Risk Factors & Measured As & Criteria \\
\hline $\mathbf{1}$ & Prevalence of smoking in Male & $\%$ of adults & Smaller is the \\
best & Smaller is the \\
best & Prevalence of smoking in Male & $\%$ of adults & best \\
\hline
\end{tabular}




\begin{tabular}{|c|c|c|c|}
\hline 3 & Incidence of tuberculosis & per 100,000 people & $\begin{array}{l}\text { Smaller is the } \\
\text { best }\end{array}$ \\
\hline 4 & Prevalence of diabetes & $\%$ of population ages 20 to 79 & $\begin{array}{l}\text { Smaller is the } \\
\text { best }\end{array}$ \\
\hline 5 & Incidence of HIV & $\begin{array}{l}\text { per } 1,000 \text { uninfected population ages } 15 \text {. } \\
49\end{array}$ & $\begin{array}{l}\text { Smaller is the } \\
\text { best }\end{array}$ \\
\hline 6 & Prevalence of HIV Total & $\%$ of population ages $15-49$ & $\begin{array}{l}\text { Smaller is the } \\
\text { best }\end{array}$ \\
\hline 7 & $\begin{array}{l}\text { Prevalence of HIV Women's share of } \\
\text { population ages } 15+\end{array}$ & $\begin{array}{l}\% \text { Women's share of population } \\
\text { ages } 15+\end{array}$ & $\begin{array}{l}\text { Smaller is the } \\
\text { best }\end{array}$ \\
\hline 8 & $\begin{array}{l}\text { Prevalence of HIV Youth, Male ages 15- } \\
24\end{array}$ & $\%$ of population ages $15-24$ & $\begin{array}{l}\text { Smaller is the } \\
\text { best }\end{array}$ \\
\hline 9 & $\begin{array}{l}\text { Prevalence of HIV Youth, Male ages 15- } \\
24\end{array}$ & $\%$ of population ages $15-24$ & $\begin{array}{l}\text { Smaller is the } \\
\text { best }\end{array}$ \\
\hline 10 & Antiretroviral therapy coverage & $\%$ of people living with HIV & $\begin{array}{l}\text { Smaller is the } \\
\text { best }\end{array}$ \\
\hline
\end{tabular}

There are ten factors representing future health risks to a country's health system. All factors possess the characteristic of smaller acceptable therefore in order to normalize the data we adopted the procedure accordingly.

\section{Methodology}

Following the quantitative research philosophy and deductive approach, overall design of the study comprises of survey of literature from the aforementioned databases, extraction of data from WDI 2020 and mathematical analysis. Secondary data of one hundred-six countries was extracted from the website of WDI 2020. The number of countries was decided on the basis of availability of data on the variables (Table 1). Classical procedure of Grey Relational Analysis (GRA) has been used as research methodology (Hamzacebi et al., 2011; Kuo et al., 2008; Tayyar et al., 2014; Wu, 2002). GRA proceed stepwise as given in Niazi et al. (2021) and Qazi et al. (2021), whereas, scheme of notations have been adopted from Ertugrul et al. (2016).

Step 1: Created a data set and established decision matrix Equation (1)

$$
x_{i}(k)=\left[\begin{array}{ccc}
x_{1}(1) x_{1}(2) & \cdots & x_{1}(m) \\
\vdots & \ddots & \vdots \\
x_{n}(1) x_{n}(2) & \cdots & x_{n}(m)
\end{array}\right]
$$

Equation (1)

Table 2: Health Risk Factors Data

\begin{tabular}{|c|c|c|c|c|c|c|c|c|c|c|c|}
\hline Sr. & Country & 1 & 2 & 3 & 4 & 5 & 6 & 7 & 8 & 9 & 10 \\
\hline 1 & Algeria & 30 & 1 & 69 & 6.7 & 0.1 & 0.1 & 46 & 0 & 0.1 & 81 \\
\hline 2 & Argentina & 28 & 16 & 27 & 5.9 & 0.3 & 0.4 & 32 & 0 & 0.1 & 61 \\
\hline$\cdots$ & ............ & $\cdots$ & ... & $\cdots$ & $\cdots$ & $\cdots$ & $\cdots$ & $\cdots$ & ... & $\cdots$ & $\cdots$ \\
\hline$\ldots$ & ............ & ... & $\ldots$ & $\ldots$ & $\ldots$ & $\ldots$ & $\ldots$ & $\ldots$ & ... & $\ldots$ & $\ldots$ \\
\hline 78 & Pakistan & 37 & 3 & 265 & 20 & 0.2 & 0.1 & 30 & 0 & 0.1 & 10 \\
\hline 79 & Panama & 10 & 2 & 52 & 7.7 & 0.6 & 0.9 & 30 & 0 & 0.1 & 54 \\
\hline$\cdots$ & ........... & $\cdots$ & $\cdots$ & $\cdots$ & $\cdots$ & $\cdots$ & $\cdots$ & $\cdots$ & $\cdots$ & $\cdots$ & $\cdots$ \\
\hline$\cdots$ & ........... & ㅇ & $\cdots$ & $\cdots$ & $\cdots$ & $\cdots$ & $\cdots$ & $\cdots$ & $\cdots$ & $\cdots$ & $\cdots$ \\
\hline 105 & Zambia & 25 & 3 & 346 & 4.5 & 5.7 & 11 & 59 & 2 & 5 & 78 \\
\hline 106 & Zimbabwe & 31 & 2 & 210 & 1.8 & 4.9 & 13 & 60 & 3 & 5.7 & 88 \\
\hline
\end{tabular}

Step 2: Created reference series and comparison matrix Equation (2)

$$
x_{0}=\left[x_{0}(k) \ldots \ldots \ldots \ldots x_{0}(n)\right] \quad \text { Equation }(2)
$$

Table 3: Reference Sequence and Comparable Sequences

\begin{tabular}{llllllllllll}
\hline Sr. & Country & 1 & 2 & 3 & 4 & 5 & 6 & 7 & 8 & 9 & 10 \\
\hline
\end{tabular}




\begin{tabular}{clcccccccccc}
\hline $\mathbf{0}$ & Reference Sequences & 8 & 0 & 0 & 1 & 0 & 0.1 & 6 & 0 & 0.1 & 92 \\
$\mathbf{1}$ & Algeria & 30 & 1 & 69 & 6.7 & 0.1 & 0.1 & 46 & 0 & 0.1 & 81 \\
$\mathbf{2}$ & Argentina & 28 & 16 & 27 & 5.9 & 0.3 & 0.4 & 32 & 0 & 0.1 & 61 \\
$\ldots$ & $\ldots \ldots \ldots \ldots$ & $\ldots$ & $\ldots$ & $\ldots$ & $\ldots$ & $\ldots$ & $\ldots$ & $\ldots$ & $\ldots$ & $\ldots$ & $\ldots$ \\
$\ldots$ & $\ldots \ldots \ldots .$. & $\ldots$ & $\ldots$ & $\ldots$ & $\ldots$ & $\ldots$ & $\ldots$ & $\ldots$ & $\ldots$ & $\ldots$ & $\ldots$ \\
$\mathbf{7 8}$ & Pakistan & 37 & 3 & 265 & 20 & 0.2 & 0.1 & 30 & 0 & 0.1 & 10 \\
$\mathbf{7 9}$ & Panama & 10 & 2 & 52 & 7.7 & 0.6 & 0.9 & 30 & 0 & 0.1 & 54 \\
$\ldots$ & $\ldots \ldots \ldots \ldots$. & $\ldots$ & $\ldots$ & $\ldots$ & $\ldots$ & $\ldots$ & $\ldots$ & $\ldots$ & $\ldots$ & $\ldots$ & $\ldots$ \\
$\ldots$ & $\ldots \ldots \ldots .$. & $\ldots$ & $\ldots$ & $\ldots$ & $\ldots$ & $\ldots$ & $\ldots$ & $\ldots$ & $\ldots$ & $\ldots$ & $\ldots$ \\
$\mathbf{1 0 5}$ & Zambia & 25 & 3 & 346 & 4.5 & 5.7 & 11 & 59 & 2 & 5 & 78 \\
$\mathbf{1 0 6}$ & Zimbabwe & 31 & 2 & 210 & 1.8 & 4.9 & 13 & 60 & 3 & 5.7 & 88 \\
\hline
\end{tabular}

Step 3: Created a normalized matrix for smaller the better Equation (3)

$$
x_{i}(k)=\frac{\max _{i}^{(o)}(k)-x_{i}^{(0)}(k)}{\max _{i}^{(0)}(k)-\min x_{i}^{(0)}(k)}
$$

Equation (3)

Table 4: Normalized Comparable Sequences

\begin{tabular}{|c|c|c|c|c|c|c|c|c|c|c|c|}
\hline Sr. & Country & 1 & 2 & 3 & 4 & 5 & 6 & 7 & 8 & 9 & 10 \\
\hline $\mathbf{0}$ & $\begin{array}{l}\text { Reference } \\
\text { Sequences }\end{array}$ & 1.0000 & 1.0000 & 1.0000 & 1.0000 & 1.0000 & 1.0000 & 1.0000 & 1.0000 & 1.0000 & 1.0000 \\
\hline 1 & Algeria & 0.6765 & 0.9773 & 0.8871 & 0.7286 & 0.9933 & 1.0000 & 0.3443 & 1.0000 & 1.0000 & 0.8659 \\
\hline 2 & Argentina & 0.7059 & 0.6364 & 0.9558 & 0.7667 & 0.9800 & 0.9888 & 0.5738 & 1.0000 & 1.0000 & 0.6220 \\
\hline$\cdots$ & …......... & $\cdots$ & $\cdots$ & $\cdots$ & $\cdots$ & $\cdots$ & $\cdots$ & $\cdots$ & $\cdots$ & $\cdots$ & $\cdots$ \\
\hline \% & & $\ldots$ & $\ddot{03} 18$ & & & $\ldots$ & & $\ddot{0}$ & $\ldots$ & $\ldots$ & $0 \ddot{0}$ \\
\hline 78 & Pakistan & 0.5735 & 0.9318 & 0.5663 & 0.0952 & 0.9867 & 1.0000 & 0.6066 & 1.0000 & 1.0000 & 0.0000 \\
\hline 79 & Panama & 0.9706 & 0.9545 & 0.9149 & 0.6810 & 0.9600 & 0.9703 & 0.6066 & 1.0000 & 1.0000 & 0.5366 \\
\hline$\cdots$ & ............ & $\cdots$ & $\cdots$ & $\cdots$ & $\cdots$ & $\cdots$ & $\cdots$ & $\cdots$ & $\cdots$ & $\cdots$ & $\cdots$ \\
\hline$\ldots$ & Zambia... & 0.7500 & $0 . \ddot{9318}$ & $0 . \ddot{4337}$ & $0 . \ddot{8333}$ & $0 . \ddot{6200}$ & 0. & 0.1311 & $0 . \cdots$ & $\begin{array}{c}\dddot{6} \\
0.6918\end{array}$ & $\begin{array}{c}\dddot{.} \\
0.8293\end{array}$ \\
\hline 106 & Zimbabwe & 0.6618 & 0.9545 & 0.6563 & 0.9619 & 0.6733 & 0.5204 & 0.1148 & 0.4000 & 0.6478 & 0.9512 \\
\hline
\end{tabular}

For instance, calculation of Algeria, 'Prevalence of smoking in Male'

$$
x_{1}^{*}(1)=\frac{\max x_{1}^{0}(1)-x_{1}^{0}(1)}{\max x_{1}^{0}(1)-\min x_{1}^{o}(1)}=\frac{76-30}{76-8}=0.6765
$$

Step 4: Obtained absolute values by calculating deviation sequence Equation (4)

$$
\Delta_{0}(\gamma)=\left|x_{0}(\gamma)-x_{1}(\gamma)\right| \quad \text { Equation (4) }
$$

Deviation Sequence is calculated Equation (5), Equation (6) and Equation (7)

$$
\Delta_{0 i}(k)=\left|x_{0}^{*}(k)-x_{i}^{*}(k)\right| \quad \text { Equation (5) }
$$

For biggest deviation:

$$
\Delta_{\max }=\underset{\forall j \varepsilon i}{\max } \max _{\forall k}\left|x_{0}^{*}(k)-x_{j}^{*}(k)\right| \quad \text { Equation (6) }
$$

\begin{tabular}{|c|c|c|c|c|c|c|c|c|c|c|c|}
\hline Sr. & Country & 1 & 2 & 3 & 4 & 5 & 6 & 7 & 8 & 9 & 10 \\
\hline 0 & $\begin{array}{l}\text { Reference } \\
\text { Sequences }\end{array}$ & 0.0000 & 0.0000 & 0.0000 & 0.0000 & 0.0000 & 0.0000 & 0.0000 & 0.0000 & 0.0000 & 0.0000 \\
\hline 1 & Algeria & 0.3235 & 0.0227 & 0.1129 & 0.2714 & 0.0067 & 0.0000 & 0.6557 & 0.0000 & 0.0000 & 0.1341 \\
\hline & & & & & 223 & & & & & & \\
\hline
\end{tabular}

For smallest deviation:

$$
\Delta_{\min }=\min _{\forall j \varepsilon i} \min _{\forall k}\left|x_{0}^{*}(k)-x_{j}^{*}(k)\right| \quad \text { Equation (7) }
$$

Table 5: Deviation Sequences 


\begin{tabular}{clcccccccccc}
$\mathbf{2}$ & Argentina & 0.2941 & 0.3636 & 0.0442 & 0.2333 & 0.0200 & 0.0112 & 0.4262 & 0.0000 & 0.0000 & 0.3780 \\
$\ldots$ & $\ldots \ldots \ldots \ldots$ & $\ldots$ & $\ldots$ & $\ldots$ & $\ldots$ & $\ldots$ & $\ldots$ & $\ldots$ & $\ldots$ & $\ldots$ & $\ldots$ \\
$\ldots$ & $\ldots \ldots \ldots$. & $\ldots$ & $\ldots$ & $\ldots$ & $\ldots$ & $\ldots$ & $\ldots$ & $\ldots$ & $\ldots$ & $\ldots$ & $\ldots$ \\
$\mathbf{7 8}$ & Pakistan & 0.4265 & 0.0682 & 0.4337 & 0.9048 & 0.0133 & 0.0000 & 0.3934 & 0.0000 & 0.0000 & 1.0000 \\
$\mathbf{7 9}$ & Panama & 0.0294 & 0.0455 & 0.0851 & 0.3190 & 0.0400 & 0.0297 & 0.3934 & 0.0000 & 0.0000 & 0.4634 \\
$\ldots$ & $\ldots \ldots \ldots$. & $\ldots$ & $\ldots$ & $\ldots$ & $\ldots$ & $\ldots$ & $\ldots$ & $\ldots$ & $\ldots$ & $\ldots$ & $\ldots$ \\
$\ldots$ & $\ldots \ldots \ldots \ldots$ & $\ldots . .$. & $\ldots$ & $\ldots$ & $\ldots$ & $\ldots$ & $\ldots$ & $\ldots$ & $\ldots$ & $\ldots$ & $\ldots$ \\
$\mathbf{1 0 5}$ & Zambia & 0.2500 & 0.0682 & 0.5663 & 0.1667 & 0.3800 & 0.4052 & 0.8689 & 0.4000 & 0.3082 & 0.1707 \\
$\mathbf{1 0 6}$ & Zimbabwe & 0.3382 & 0.0455 & 0.3437 & 0.0381 & 0.3267 & 0.4796 & 0.8852 & 0.6000 & 0.3522 & 0.0488 \\
\hline
\end{tabular}

For instance, calculation of deviation of Argentina for 'Prevalence of smoking in Male'

$$
\triangle_{02}(2)=\left|x_{0}^{*}(2)-x_{2}^{*}(2)\right|=|1-0.6364|=0.3636
$$

Step 5: Grey relational co-efficient is calculated Equation (8). Term $\xi$ is distinguishing co-efficient between 0 and 1 the value of which is 0.5 in literature.

$$
\gamma\left[x_{0}^{*}(k), x_{i}^{*}(k)\right]=\frac{\Delta_{\min }+\xi \Delta_{\max }}{x_{0 i}(k)+\xi \Delta_{\max }}, 0<\gamma\left[x_{o}^{*}(k), x_{i}^{*}(k)\right] \leq 1 \quad \text { Equation (8) }
$$

\begin{tabular}{|c|c|c|c|c|c|c|c|c|c|c|c|}
\hline Sr. & \begin{tabular}{|l} 
Country \\
\end{tabular} & 1 & 2 & 3 & 4 & 5 & 6 & 7 & 8 & 9 & 10 \\
\hline $\mathbf{0}$ & $\begin{array}{l}\text { Reference } \\
\text { Sequences }\end{array}$ & 1.0000 & 1.0000 & 1.0000 & 1.0000 & 1.0000 & 1.0000 & 1.0000 & 1.0000 & 1.0000 & 1.0000 \\
\hline 1 & Algeria & 0.6071 & 0.9565 & 0.8158 & 0.6481 & 0.9868 & 1.0000 & 0.4326 & 1.0000 & 1.0000 & 0.7885 \\
\hline 2 & Argentina & 0.6296 & 0.5789 & 0.9188 & 0.6818 & 0.9615 & 0.9782 & 0.5398 & 1.0000 & 1.0000 & 0.5694 \\
\hline$\cdots$ & …......... & ... & $\cdots$ & $\cdots$ & $\cdots$ & $\cdots$ & $\cdots$ & ... & $\cdots$ & $\cdots$ & $\cdots$ \\
\hline$\ldots$ & ............ & $\ddot{0}$ & 푸 & $\ddot{0} 255$ & $\ddot{2550}$ & ‥ & ‥ & $\ddot{0}$ & ‥ & ‥ & $\ddot{2}$ \\
\hline 78 & Pakistan & 0.5397 & 0.8800 & 0.5355 & 0.3559 & 0.9740 & 1.0000 & 0.5596 & 1.0000 & 1.0000 & 0.3333 \\
\hline 79 & Panama & 0.9444 & 0.9167 & 0.8545 & 0.6105 & 0.9259 & 0.9439 & 0.5596 & 1.0000 & 1.0000 & 0.5190 \\
\hline$\cdots$ & ............. & $\cdots$ & $\cdots$ & $\cdots$ & $\cdots$ & $\cdots$ & $\cdots$ & $\cdots$ & $\cdots$ & $\cdots$ & $\cdots$ \\
\hline$\ddot{105}$ & Zambia & $0 . \ddot{6} 667$ & $0 . \ddot{8800}$ & $0 . \ddot{4689}$ & $0 . \ddot{7500}$ & $0 . \ddot{5682}$ & $0 . \ddot{5524}$ & $0 . \ddot{3653}$ & $0 . \ddot{5556}$ & $0 . \ddot{6} 187$ & $0 . \ddot{7455}$ \\
\hline 106 & Zimbabwe & 0.5965 & 0.9167 & 0.5926 & 0.9292 & 0.6048 & 0.5104 & 0.3609 & 0.4545 & 0.5867 & 0.9111 \\
\hline
\end{tabular}

Table 6: Grey Relational Co-efficient

For instance, calculation of grey relational co-efficient of Argentina for 'Prevalence of smoking in Male'

$$
\gamma\left[x_{0}^{*}(2), x_{2}^{*}(2)\right]=\frac{\Delta_{\min +\xi} \Delta_{\max }}{\Delta_{2}(2)+\xi \Delta_{\max }}=\frac{0+(0.5) \times 1}{0.3636+(0.5) \times 1}=0.5789
$$

Step 6: Weighted sum of grey relational co-efficient (Grey Relational Grade) Equation (9) and Equation (10)

\begin{tabular}{|c|c|c|}
\hline Sr. & Country & Grey Relational Grades \\
\hline $\mathbf{0}$ & Reference Sequences & 1.0000 \\
\hline 1 & Algeria & 0.8235 \\
\hline 2 & Argentina & 0.7858 \\
\hline$\cdots$ & ............ & $\cdots$ \\
\hline$\ldots$ & ............ & $\cdots$ \\
\hline 78 & Pakistan & 0.7178 \\
\hline 79 & Panama & 0.8275 \\
\hline ... & ............ & $\cdots$ \\
\hline$\ldots$ & ........... & $\ldots$ \\
\hline 105 & Zambia & 0.6171 \\
\hline 106 & Zimbabwe & 0.6464 \\
\hline
\end{tabular}

$$
\begin{array}{cc}
\gamma\left(x_{0}^{*}, x_{i}^{*}\right)=\sum_{k=1}^{n} \beta_{k} \gamma\left[x_{0}^{*}(k), x_{i}^{*}(k)\right] & \text { Equation (9) } \\
\sum_{k=1}^{n} \beta_{k}=1 & \text { Equation (10) }
\end{array}
$$

Table 7: Grey Relational Grades

For instance, grey relational grade for Argentina is calculated: 


$$
\begin{gathered}
\gamma\left(x_{0}^{*}, x_{2}^{*}\right)=\sum_{k=1}^{n} \beta_{k} \gamma\left[x_{0}^{*}(2), x_{2}^{*}(k)\right] \\
=0.10 \times(0.6296+0.5789+0.9188+0.6818+0.9615+0.9782+0.5398+1.0000+1.0000 \\
+0.5694)=0.7858
\end{gathered}
$$

\section{Results \& Discussion}

With the aim of analyzing the health risk factors of 106 countries and using a country level secondary data of ten variables taken from website of WDI 2020, the study applied Grey Relational Analysis (GRA). The study generated grey relational grades (Table 7), the same have been represented below as Table 8 into a classified form. One hundred-six countries have been divided on an ordinal scale of seven items i.e. exceptionally high, very high, high, about the same, low, very low and exceptionally low. Where the exceptionally high means that the countries have high performing health systems, therefore, there is exceptionally low level of future health risks, whereas, exceptionally low means that these countries have exceptionally low performance

\begin{tabular}{|c|c|c|c|c|c|c|c|c|}
\hline Country & GRGs & Rank & Country & GRGs & Rank & Country & GRGs & Rank \\
\hline $\begin{array}{l}\text { Reference } \\
\text { Sequences }\end{array}$ & 1 & 0 & Germany & 0.7978 & 35 & Bulgaria & 0.7607 & 71 \\
\hline \multicolumn{3}{|c|}{ Exceptionally High } & Czech Republic & 0.7974 & 36 & Liberia & 0.7604 & 72 \\
\hline Australia & 0.8735 & 1 & Kuwait & 0.7972 & 37 & Nigeria & 0.7599 & 73 \\
\hline Cabo Verde & 0.8692 & 2 & Slovak Republic & 0.7968 & 38 & Romania & 0.7599 & 74 \\
\hline Singapore & 0.862 & 3 & Ghana & 0.796 & 39 & Montenegro & 0.7577 & 75 \\
\hline Japan & 0.8543 & 4 & Armenia & 0.7947 & 40 & \multicolumn{3}{|c|}{ Very Low } \\
\hline Colombia & 0.851 & 5 & El Salvador & 0.7934 & 41 & Bahamas, The & 0.7558 & 76 \\
\hline Iceland & 0.8467 & 6 & Yemen, Rep. & 0.7929 & 42 & Moldova & 0.7536 & 77 \\
\hline New Zealand & 0.8455 & 7 & Burkina Faso & 0.7915 & 43 & Mongolia & 0.7514 & 78 \\
\hline Benin & 0.8449 & 8 & Hungary & 0.7913 & 44 & Nepal & 0.7509 & 79 \\
\hline Ecuador & 0.8437 & 9 & Uruguay & 0.7896 & 45 & Gambia & 0.7491 & 80 \\
\hline Denmark & 0.8389 & 10 & \multicolumn{3}{|c|}{ About the Same } & Latvia & 0.748 & 81 \\
\hline Niger & 0.8385 & 11 & Morocco & 0.7875 & 46 & Bangladesh & 0.7451 & 82 \\
\hline Italy & 0.8363 & 12 & Paraguay & 0.786 & 47 & Lao PDR & 0.7444 & 83 \\
\hline Netherlands & 0.8343 & 13 & Argentina & 0.7858 & 48 & Ukraine & 0.7408 & 84 \\
\hline Norway & 0.8329 & 14 & Cuba & 0.7851 & 49 & Djibouti & 0.7379 & 85 \\
\hline Panama & 0.8275 & 15 & Vietnam & 0.7838 & 50 & Mauritius & 0.7367 & 86 \\
\hline Very & igh & & Togo & 0.7834 & 51 & Suriname & 0.7356 & 87 \\
\hline Oman & 0.827 & 16 & Thailand & 0.7787 & 52 & Jamaica & 0.7248 & 88 \\
\hline Senegal & 0.8266 & 17 & Cambodia & 0.7787 & 53 & Pakistan & 0.7178 & 89 \\
\hline Mexico & 0.8242 & 18 & Malaysia & 0.7778 & 54 & Indonesia & 0.7154 & 90 \\
\hline Algeria & 0.8235 & 19 & Tunisia & 0.7773 & 55 & \multicolumn{3}{|c|}{ Exceptionally Low } \\
\hline Ireland & 0.8222 & 20 & $\begin{array}{l}\text { Bosnia and } \\
\text { Herzegovina }\end{array}$ & 0.7768 & 56 & Haiti & 0.7124 & 91 \\
\hline Costa Rica & 0.821 & 21 & Egypt, Arab Rep. & 0.7754 & 57 & Uganda & 0.7093 & 92 \\
\hline Finland & 0.821 & 22 & Mali & 0.7754 & 58 & Kenya & 0.6913 & 93 \\
\hline Ethiopia & 0.8203 & 23 & Serbia & 0.7753 & 59 & Sierra Leone & 0.6809 & 94 \\
\hline Spain & 0.8184 & 24 & Kazakhstan & 0.7752 & 60 & Tanzania & 0.6786 & 95 \\
\hline Eritrea & 0.8176 & 25 & \multicolumn{3}{|c|}{ Low } & $\begin{array}{l}\text { Papua New } \\
\text { Guinea }\end{array}$ & 0.6576 & 96 \\
\hline Croatia & 0.8153 & 26 & Lebanon & 0.7749 & 61 & Zimbabwe & 0.6464 & 97 \\
\hline Iran, Islamic Rep. & 0.8152 & 27 & Rwanda & 0.7748 & 62 & Congo, Rep. & 0.6454 & 98 \\
\hline Portugal & 0.8139 & 28 & Dominican Republic & 0.7733 & 63 & Malawi & 0.6434 & 99 \\
\hline Luxembourg & 0.8137 & 29 & Belarus & 0.7697 & 64 & Zambia & 0.6171 & 100 \\
\hline Comoros & 0.8116 & 30 & Georgia & 0.7676 & 65 & Namibia & 0.5967 & 101 \\
\hline \multicolumn{3}{|c|}{ High } & Estonia & 0.7665 & 66 & Eswatini & 0.5614 & 102 \\
\hline Uzbekistan & 0.8081 & 31 & Philippines & 0.7663 & 67 & Botswana & 0.5474 & 103 \\
\hline France & 0.806 & 32 & Chile & 0.7647 & 68 & Mozambique & 0.5421 & 104 \\
\hline
\end{tabular}
health systems, therefore, are exposed to high level of future health risks.

Table 8: Results of Grey Relational Analysis 


\begin{tabular}{lll|lll|lll} 
Sri Lanka & 0.8006 & 33 & Kyrgyz Republic & 0.7629 & 69 & Lesotho & 0.4975 & 105 \\
Barbados & 0.7993 & 34 & Myanmar & 0.7628 & 70 & South Africa & 0.4749 & 106 \\
\hline *GRG - Grey Relational Grades
\end{tabular}

Most of the countries having exceptionally high performance on health systems (exposed to low future health risks) are member countries of Organization for Economic Co-operation and Development (OECD). Member countries of European Union (EU) and OECD also have very high performance health systems and are relatively less exposed to future health risks. Member countries of Arabian Countries (AC), Association of Southeast Asian Nations (ASEAN) and Japan \& Asian Pacific Region (J\&APR) are expected to remain at same level of future health risks. Eastern Europe (EE) countries have low level performance on health systems and are exposed to relatively high level of future health risks. Member countries of Caribbean Community and Common Market (CARICOM) and South Asian Association for Regional Cooperation (SAARC) have very low performance on health systems and they are expected to be exposed very high level of future health risks. Member countries of Southern Africa Development Community (SADC) have exceptionally low performance on health systems and they are exposed to exceptionally high level of future health risks. Right form the aim of the study up to the analysis and results, it is a different study. The study is different in design, data sets, scope (number of countries), methodology, analysis and results. It is also different from contemporary literature in terms of information, contribution, implications and impact. However, the results of the study are aligned with contemporary literature and seems to be logically valid qua reality. The study used original reliable secondary data set and concluded on the basis of mathematical procedure applied thereon to data that as a result provide additional information and a framework for future researchers.

\section{Conclusion}

Health system of a country is considered to be one of the core systems of economy. It has fundamental importance to economic development. It is an ever green area of research. Country level comparative studies of health system remained always relevant and presently they have become more pertinent. Countries are always considered to evaluate the future health risks and their preparedness to cop them up. Therefore, aim of this study is to evaluate the country level positions of 106 countries and compare them on objective basis for their exposition the future health risks. The data of countries was extracted from WDI 2020 for ten future health risks variables. A composite mathematical analysis is performed through Grey Relational Analysis (GRA). Results of GRA show that most of the countries having exceptionally high performance on health systems (exposed to low future health risks) are member countries of OECD. Member countries of EU and OECD also have very high performance health systems and are relatively less exposed to future health risks. Member countries of AC, ASEAN) and J\&APR are expected to remain at same level of future health risks. EE countries have low level performance on health systems and are exposed to relatively high level of future health risks. Member countries of CARICOM and SAARC have very low performance on health systems and they are expected to be exposed very high level of future health risks. Member countries of SADC have exceptionally low performance on health systems and they are exposed to exceptionally high level of future health risks. The study has significant theoretical, practical and policy implications. The study contributed grey relational grades and grey relational co-efficient along with country classifications towards theoretical literature of the domain. It provides the theoretical frameworks for the future researchers along with lot of additional information. It also provides insights to policy makers to evaluate their positions before embarking on some future discourse to mitigate the future health risks. It also provides guideline to political government to readjust their positions as against their rivals. The study also has certain limitations, it is a cross sectional study, therefore, the results can only be generalized accordingly, longitudinal studies are recommended for future. The study uses secondary data in a composite mathematical 
methodology i.e. GRA, whereas there is a wide array of statistical methodologies that can be verify the results of GRA. All the variables have been given equal weights, the results might change if inter-variable weights are change by way of Entropy method or expert opinion.

\section{References}

Aghdam, Z. N., Rahmani, A. M., \& Hosseinzadeh, M. (2020). The Role of the Internet of Things in Healthcare: Future Trends and Challenges. Computer methods and programs in biomedicine, 105903.

Amariles, P., Ledezma-Morales, M., Salazar-Ospina, A., \& Hincapié-García, J.A. (2020). How to link patients with suspicious COVID-19 to health system from the community pharmacies? A route proposal. Research in Social and Administrative Pharmacy, 17(1), 1988-1989.

Armocida, B., Formenti, B., Ussai, S., Palestra, F., \& Missoni, E. (2020). The Italian health system and the COVID-19 challenge. The Lancet Public Health, 5(5), e253.

Bolnick, H. J., Bui, A. L., Bulchis, A., Chen, C., Chapin, A., Lomsadze, L., ... \& Dieleman, J. L. (2020). Health-care spending attributable to modifiable risk factors in the USA: an economic attribution analysis. The Lancet Public Health, 5(10), e525-e535.

Briggs, A. M., Shiffman, J., Shawar, Y. R., Åkesson, K., Ali, N., \& Woolf, A. D. (2020). Global health policy in the 21st century: Challenges and opportunities to arrest the global disability burden from musculoskeletal health conditions. Best Practice \& Research Clinical Rheumatology, 101549.

Buckley, A., Bianco, A., \& Stone, J. (2020). Universal testing of patients and their support persons for COVID-19 when presenting for admission to Labor and Delivery within the Mount Sinai Health System. American Journal of Obstetrics \& Gynecology MFM, 100147.

Chen, T., Wang, Y., \& Hua, L. (2020). "Pairing assistance": The effective way to solve the breakdown of health services system caused by COVID-19 pandemic. International Journal for Equity in Health, 19(1), 1-4.

Cheng, Y., Nathanail, C. P., \& Ja'afaru, S. W. (2019). Generic assessment criteria for human health risk management of agricultural land scenario in Jiangsu Province, China. Science of The Total Environment, 697, 134071.

Dahler-Larsen, P., Sundby, A., \& Boodhoo, A. (2020). Can occupational health and safety management systems address psychosocial risk factors? An empirical study. Safety Science, 130, 104878.

Dash, S., Shakyawar, S.K., Sharma, M. (2019). Big data in healthcare: management, analysis and future prospects. Journal of Big Data 6, 54.

de León-Martínez, L. D., Palacios-Ramírez, A., Rodriguez-Aguilar, M., \& Flores-Ramírez, R. (2020). Critical review of social, environmental and health risk factors in the Mexican indigenous population and their capacity to respond to the COVID-19. Science of The Total Environment, 733, 139357.

Dzhafer, N., \& Papathanasiou, J. (2020). Compassionate drug use: an imperative challenge for Bulgarian health system during COVID-19. Health Policy and Technology, 9(3), 274-275.

Ertugrul, I., Oztas, T., Ozcil, A., \& Oztas, G. Z. (2016). Grey relational analysis approach in academic performance comparison of university: A case study of Turkish universities. European Scientific Journal, 7881, 128-139.

Gao, D., Ripley, S., Weichenthal, S., \& Pollitt, K. J. G. (2020). Ambient particulate matter oxidative potential: Chemical determinants, associated health effects, and strategies for risk management. Free Radical Biology and Medicine, 151, 7-25.

Hamzaçebi, C., \& Pekkaya, M. (2011). Determining of stock investments with grey relational analysis. Expert Systems with Applications, 38(8), 9186-9195.

Hsieh, V.C.R. (2020). Putting resiliency of a health system to the test: COVID-19 in Taiwan. Journal of the Formosan Medical Association, 119, 884-885.

Kapetanakis, E.I., Tomos, I. P., Karakatsani, A., Koumarianou, A., \& Tomos, P.I. (2020). Management of surgical lung cancer patients during the COVID-19 pandemic in the 
financially and resource strained Greek health care system, J Surg Oncol, 1-4.

Kuo, Y., Yang, T., \& Huang, G. W. (2008). The use of grey relational analysis in solving multiple attribute decision-making problems. Computers \& industrial engineering, 55(1), 80-93.

Legido-Quigley, H., Mateos-García, J. T., Campos, V. R., Gea-Sánchez, M., Muntaner, C., \& McKee, M. (2020). The resilience of the Spanish health system against the COVID-19 pandemic. The lancet public health, 5(5), e251-e252.

Mhango, M., Dzobo, M., Chitungo, I., \& Dzinamarira, T. (2020). COVID-19 risk factors among health workers: a rapid review. Safety and health at work, 11, 262-265.

Mussard, S., \& Alperin, M. N. P. (2021). Accounting for risk factors on health outcomes: The case of Luxembourg. European Journal of Operational Research, 291(3), 1180-1197.

Najera, H., \& Ortega-Avila, A. G. (2020). Health and Institutional Risk Factors of COVID-19 Mortality in Mexico, 2020. American Journal of Preventive Medicine.

Navarro, J.C., Arrivillaga-Henríquez, J., Salazar-Loor, J., \& Rodriguez-Morales, A.J. (2020). COVID-19 and dengue, co-epidemics in Ecuador and other countries in Latin America: Pushing strained health care systems over the edge. Travel Medicine and Infectious Disease, 37, 101656.

Niazi, A. A. K., Qazi, T. F., Basit, A.\&Shaukat, M. Z. (2021). Evaluation of Climate of Selected Sixty-six Countries using Grey Relational Analysis: Focus on Pakistan. Journal of Business and Social Review in Emerging Economies, 7(1), 51-62

Qazi, T. F., Niazi, A. A. K., Asghar, W.\&Basit, A.(2021). Ease of Doing Business: Analysis of Trade Facilitations of One Hundred Twenty-Seven Countries of the World. Journal of Accounting and Finance in Emerging Economies, 7(1), 65-75.

Queralt-Tomas, L., Clua-Espuny, J. L., Fernández-Saez, J., Lleixà-Fortuño, M. M., AlbiolZaragoza, I., Gil-Guillen, V., \& Carratala-Munuera, C. (2019). Risk of dependency: a challenge for health and social care planning-observational stroke cohort. Value in Health, 22(10), 1083-1091.

Ramos, D., Afonso, P., \& Rodrigues, M. A. (2020). Integrated management systems as a key facilitator of occupational health and safety risk management: A case study in a medium sized waste management firm. Journal of Cleaner Production, 262, 121346.

Requia, W.J., Kondo, E.K., Adams, M.D., Gold, D.R., \& Struchiner, C.J. (2020). Risk of the Brazilian health care system over 5572 municipalities to exceed health care capacity due to the 2019 novel coronavirus (COVID-19). Science of The Total Environment, 139144.

Roder-DeWan, S. (2020). Health system quality in the time of COVID-19. The Lancet Global Health, 8(6), 738-739.

Rohat, G., Flacke, J., Dosio, A., Pedde, S., Dao, H., \& van Maarseveen, M. (2019). Influence of changes in socioeconomic and climatic conditions on future heat-related health challenges in Europe. Global and planetary change, 172, 45-59.

Scharpf, F., Kaltenbach, E., Nickerson, A., \& Hecker, T. (2020). A systematic review of socioecological factors contributing to risk and protection of the mental health of refugee children and adolescents. Clinical Psychology Review, 83, 101930.

Tayyar, N., Akcanlı, F., Genç, E., \& Erem, I. (2014). Evaluating the financial performance of companies operating in the field of informatics and technology registered in BIST by analytical hierarchy process (AHP) and gray relational analysis (TIA) method. Accounting and Finance Journal, 61, 19-40.

World Development Indicators. (2020). Retrieved April 15, 2020, from http://wdi.worldbank.org/tables.

$\mathrm{Wu}, \mathrm{H} . \mathrm{H}$. (2002). A comparative study of using grey relational analysis in multiple attribute decision making problems. Quality Engineering, 15(2), 209-217.

Xu, X., Hu, X., Wang, T., Sun, M., Wang, L., \& Zhang, L. (2021). Non-inverted U-shaped challenges to regional sustainability: The health risk of soil heavy metals in coastal China. Journal of Cleaner Production, 279, 123746. 
Yadav, D., Rangabhashiyam, S., Verma, P., Singh, P., Devi, P., Kumar, P., ... \& Kumar, K. S. (2021). Environmental and Health Impacts of Contaminants of Emerging Concerns: Recent Treatment Challenges and Approaches. Chemosphere, 129492.

Yamakawa, M., Tanaka, Y., \& Sasai, M. (2019). Health risk management behaviors and related factors among Japanese university students participating in short-term study abroad programs. Journal of Infection and Chemotherapy, 25(11), 866-872.

Yang, Xiaoyi, Hongwei Li, Na Zhang, Hui Zhao, and Ruipeng Tong (2020). Evaluation and comparison of the Chinese policy context for safety-related psychological health in the workplace: Realities, gaps and challenges. Journal of Loss Prevention in the Process Industries, 67, 104217.

Zaidi, S. A. H., Shahbaz, M., Hou, F., \& Abbas, Q. (2021). Sustainability challenges in public health sector procurement: an application of interpretative structural modelling. SocioEconomic Planning Sciences, 101028.

Zhang, X., \& Mohandes, S. R. (2020). Occupational Health and Safety in green building construction projects: A holistic Z-numbers-based risk management framework. Journal of Cleaner Production, 275, 122788. 
